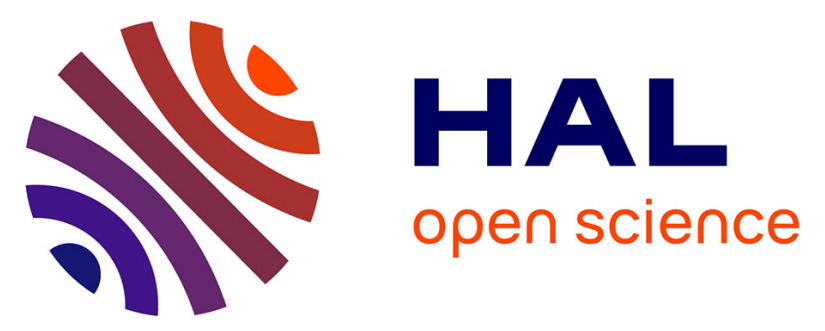

\title{
High integrity lane level localization using multiple lane markings detection and horizontal protection levels
}

\author{
Gabriel Frisch, Philippe Xu, Emmanuel Stawiarski
}

\section{To cite this version:}

Gabriel Frisch, Philippe Xu, Emmanuel Stawiarski. High integrity lane level localization using multiple lane markings detection and horizontal protection levels. 15th International Conference on Control, Automation, Robotics and Vision (ICARCV 2018), Nov 2018, Singapore, Singapore. pp.1496-1501, 10.1109/ICARCV.2018.8581278 . hal-01971384

\section{HAL Id: hal-01971384 \\ https://hal.science/hal-01971384}

Submitted on 7 Jan 2019

HAL is a multi-disciplinary open access archive for the deposit and dissemination of scientific research documents, whether they are published or not. The documents may come from teaching and research institutions in France or abroad, or from public or private research centers.
L'archive ouverte pluridisciplinaire HAL, est destinée au dépôt et à la diffusion de documents scientifiques de niveau recherche, publiés ou non, émanant des établissements d'enseignement et de recherche français ou étrangers, des laboratoires publics ou privés. 


\title{
High integrity lane level localization using multiple lane markings detection and horizontal protection levels
}

\author{
Gabriel Frisch, Philippe Xu and Emmanuel Stawiarski
}

\begin{abstract}
For autonomous driving, lane level accurate localization is a necessity for complex driving maneuvers. Classical GNSS based methods are usually not accurate enough to have an unambiguous lane level localization. Having camera measurements such as lane marking detections along with high definition maps can enhance localization performance. In this paper, we are interested in high integrity localization, meaning being robust with low risk level. We propose a novel geometrical approach using horizontal protection levels on localization to propagate uncertainties and use lane markings to have an unambiguous map-matching. We demonstrate on real data that the algorithm can cope with high levels of noise on both localization and detection.
\end{abstract}

\section{INTRODUCTION}

Having a precise and reliable pose estimation is critical for autonomous vehicles. GNSS only solutions are most of the time not precise enough to realize complex maneuvers with low risk. Many other sensors may be combined to improve this localization. High definition maps can also be used to improve accuracy. Maps contain a lot of information such as positions of traffic signs, intersections, lane markings or driving lanes [3], [4]. In order to use information from maps, it is necessary to realize a map-matching procedure, which means making a link between the objects detected by the sensors and the features in the map. There exists many techniques for map-matching, with their advantages and complexity. The most simplistic ones rely on geometrical analyses, such as point-to-point or point-to-curve matching [16]. The most advanced ones use more refined concepts such as the theory of belief functions [8], Extended Kalman Filter [13] or Particle Filtering [7].

Apart from having good pose estimation, guaranteeing the reliability of the estimation is even more important and challenging in safety-critical applications such as autonomous driving. The concepts of integrity and protection levels initial defined in aviation have been extended to road vehicles in the recent years [6], [10].

The motivation of this paper is not to improve the localization but to present a method that realizes a lane level mapmatching with a high integrity. This approach uses horizontal protection levels on localization to propagate uncertainties and uses multiple lane marking detections to have a unique unambiguous map-matching.

G. Frisch and $\mathrm{Ph} . \mathrm{Xu}$ are with Sorbonne universités, Université de technologie de Compiègne, CNRS, Heudiasyc UMR 7253, 60203 Compiègne Cedex, France.

E. Stawiarski is with Renault s.a.s, Guyancourt, France.

This research has been carried out in the scope of the SIVALab laboratory shared between Renault, UTC and CNRS.

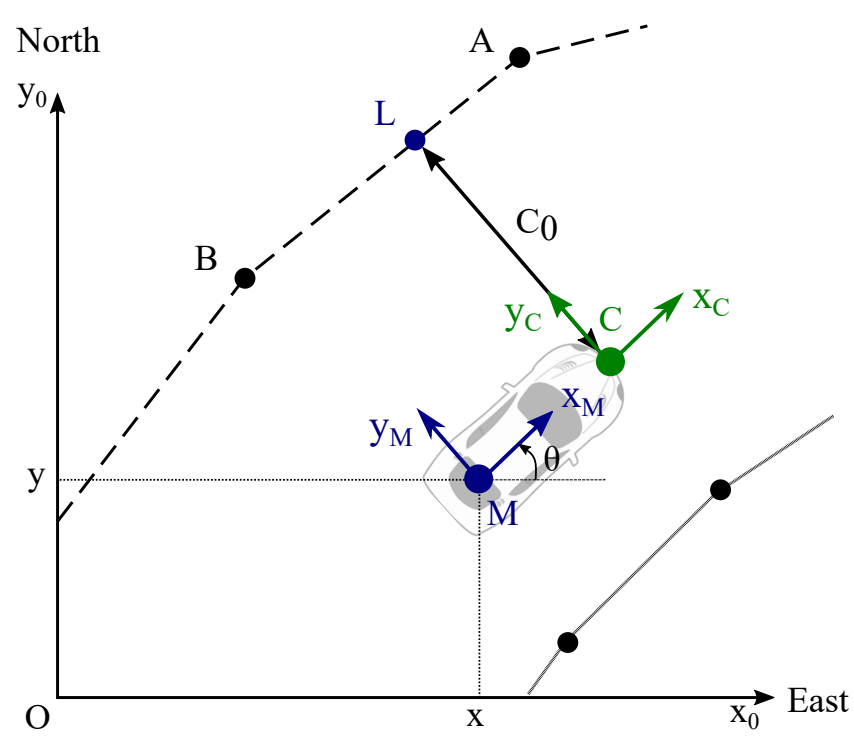

Fig. 1. Reference frames. The vehicle body frame $R_{M}$ is located at point $M$ and the camera frame $R_{C}$ is located at point $C . C_{0}$ represents the lateral distance between the camera frame and a lane marking $[A B]$.

Section II states the general problem, the map representation, the camera model and the working frames. Pose integrity concepts and over-bounding uncertainties on sensors are introduced in Section III. Section IV provides a description of the new map-matching algorithm and the multiple hypothesis disambiguation process. Finally, experimental results of this algorithm are presented in Section V.

\section{Problem statement}

The aim of this work is to position a vehicle in its actual driving lane. If the vehicle is represented by its pose, i.e., position and heading, we want to guarantee with a high degree of confidence that the coordinates of the position are within the bounds of its corresponding lane, which are assumed to be given within a map. In order to guarantee a low risk of wrong lane selection, our approach will provide a decision in case of non-ambiguous hypothesis. The uncertainty of the pose is modeled by protection levels representing a space domain in which the vehicle is certain to be with a given risk.

\section{A. Working frames}

In general, GNSS and map data are expressed in geographic coordinates [9]. However, Cartesian coordinates are usually preferred as the computation of geometric transformation becomes easier. We choose to work in a local 
Cartesian ENU (East, North, Up) [12] reference frame $R_{O}$; its origin can be any position close to the navigation area.

Several working frames are defined and illustrated in Fig. 1. The first one denoted $R_{M}$ is attached to the vehicle at point $M$, representing the middle of the rear wheels. The vehicle pose is defined at point $M$ as $[x, y, \theta]^{T}$ in $R_{O}$ which includes the position $[x, y]^{T}$ and the heading $\theta$. The second frame $R_{C}$ represents the camera frame in which the measurements will be represented. Its origin $C$ is virtually situated at the center of the front bumper. Both $x_{M}$ and $x_{C}$ are longitudinal axes pointing forward while $y_{M}$ and $y_{C}$ are lateral axes pointing to the left side of the vehicle.

\section{B. Map representation}

The map used in this research has a scale between the macro-scale (e.g., road guidance maps) and micro-scale (e.g., point cloud from perception sensors) [8]. In our map, the lanes are discretized as polylines, i.e., a sequence of line segments representing the center of the lanes. The border of a lane corresponds either to a lane marking, or the limit of the drivable road space defined by sidewalks, for example. The lane borders are also represented by polylines but with an additional associated type: solid, dashed, double, etc.

\section{Camera model}

The aim of the camera module is to return measurements of detected lane markings. The image processing step is out of the scope of this paper. In our case, we used an off-theshelf intelligent camera: Mobileye. The camera is able to detect up to four markings at a time; two on each side of the vehicle. For each detected lane marking, the coefficients of a third degree polynomial are returned. In the camera frame $R_{C}$, the equation can be expressed as

$$
y=C_{3} x^{3}+C_{2} x^{2}+C_{1} x+C_{0} .
$$

In this paper, only the coefficient $C_{0}$ is used in the mapmatching process. The value of $C_{0}$ corresponds to the lateral distance to an observed lane marking along the $y_{C}$ axis. In the example pictured in Fig. 1, the coordinates of the point $L$, which belongs to an observed lane marking $[A B]$, are equal to $\left[0, C_{0}\right]^{T}$ in the camera frame $R_{C}$. In the rest of the paper, a detected lane marking will be represented by its corresponding point $L$ in the camera frame. In addition to the lateral distance, the camera is able to give the type (dashed, continuous, double, etc.) and also a quality indicator (with value in $\{0,1,2,3\}$ ) associated with each lane marking detection. Our approach can be used with any other lane marking detection algorithm from the literature as long as they provide similar information.

\section{UNCERTAINTY AND INTEGRITY}

The uncertainty of measurements are usually represented as an additive Gaussian white noise from which probabilistic confidence bounds can be computed given a level of risk $\alpha$. Assuming that errors are normally distributed is often unrealistic in practice due to outliers [2]. Robust methods have been proposed using more cautious models such as intervals [1], [14] or heavy tail distributions [2], [5]. The aim of these methods is to over-bound the true error distribution resulting in more consistent confidence bounds called protection levels. For a given risk $\alpha$, also referred to Integrity Risk (IR), the Protection Level (PL) is a statistical bound on the Position Error (PE) such that the probability that the error exceeds the PL should be equal to the Integrity Risk: $\operatorname{Pr}(P E>P L)=I R$. Because the true probability density function of the position error is usually not known, we often handle instead a Target Integrity Risk (TIR) which verifies $\operatorname{Pr}(P E>P L) \leq T I R$.

\section{A. Pose uncertainty}

We will directly introduce the pose error within the mobile frame $R_{M}$. In this frame, the true vehicle pose is $[0,0,0]^{T}$, the estimated pose can be written as

$$
\left[\hat{x}_{M}, \hat{y}_{M}, \hat{\theta}_{M}\right]^{T}=[0,0,0]^{T}+\left[e_{x}, e_{y}, e_{\theta}\right]^{T}
$$

where $\hat{X}_{M}=\left[\hat{x}_{M}, \hat{y}_{M}, \hat{\theta}_{M}\right]^{T}$ is the estimated pose and is equal to the estimation error $\hat{e}=\left[e_{x}, e_{y}, e_{\theta}\right]^{T}$.

In general, a confidence domain $D$ at risk $\alpha$, verifies $P(\hat{e} \notin D)=\alpha$ and is defined as a 3-dimensional ellipsoid. Manipulating such domain may be complex. One solution is to over-bound it by the smallest 3-dimensional parallelepiped $\bar{D}$ aligned with the frame axis. As $D \subset \bar{D}$, we have $P(\hat{e} \notin \bar{D}) \leq \alpha$. In the case where the estimation error $\hat{e}$ follows a zero mean Gaussian distribution with a diagonal covariance matrix $\Sigma=\operatorname{diag}\left(\sigma_{x}^{2}, \sigma_{y}^{2}, \sigma_{\theta}^{2}\right)$, the domain $\bar{D}$ is defined by intervals over the three coordinates.

$$
\bar{D}=[-\bar{x},+\bar{x}] \times[-\bar{y},+\bar{y}] \times[-\bar{\theta},+\bar{\theta}],
$$

with

$$
\bar{q}=\frac{z_{1-\alpha / 2}}{\sigma_{q}}, \quad q \in\{x, y, \theta\},
$$

where $z_{\beta}$ is the $\beta$-quantile of the standard normal distribution. Within the mobile frame, $\bar{x}$ represents the along protection level $\left(P L_{x}\right), \bar{y}$ the across protection level $\left(P L_{y}\right)$ and $\bar{\theta}$ the heading protection level $\left(P L_{\theta}\right)$.

\section{B. Map and camera uncertainty}

We do not consider the map as being perfect and we decide to take into account the map positional error. As the error distribution is not known, we prefer to over-bound the error. We assume that the true position of any point defining the polylines of the map is located within a circle of radius $l$ [13]. We consider that the circle is a guaranteed domain, i.e., with a probability of one. In the same way as we did with the pose uncertainty, we bound a line segment between two consecutive points by the smallest rectangle containing the two circles as illustrated in Fig. 2.

Similarly to map uncertainties, we also over-bound the camera measurement error within a $\pm \Delta C_{0}$ bound. If the uncertainties on maps and camera measurements are known, these bounds can also be replaced by confidence domains w.r.t. a given risk. These uncertainties are illustrated in Fig. 2. 


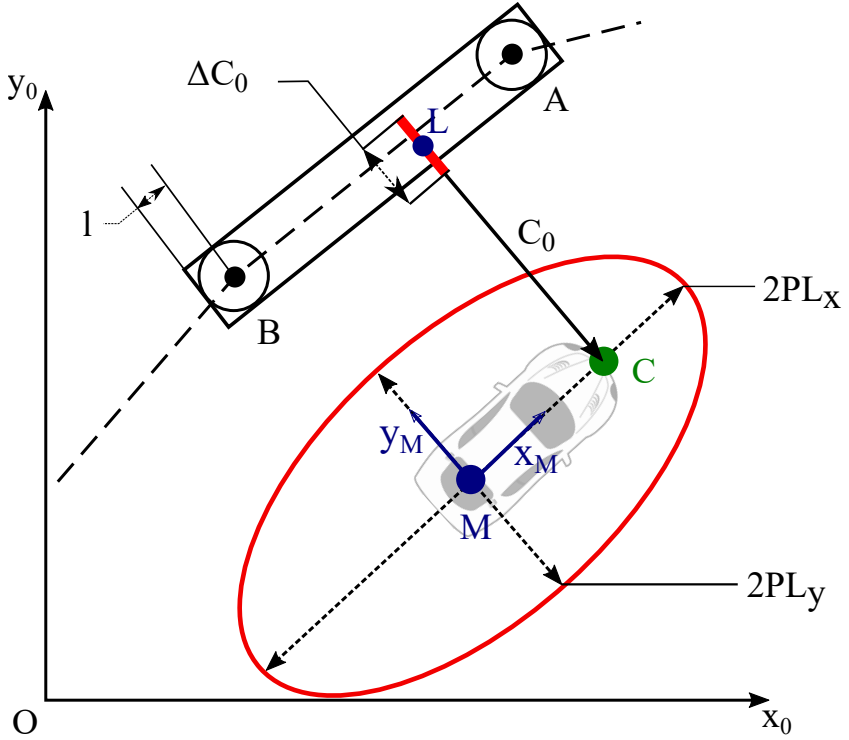

Fig. 2. Representation of map, camera and localization uncertainties. Points $\mathrm{A}$ and $\mathrm{B}$ are supposed to be within a circle of radius $l$. The lateral distance of the detected marking is $C_{0}$ with its uncertainty $\Delta C_{0}$.

\section{MAP MATChing OF THE CAMERA MEASUREMEnTS}

\section{A. Principle of the algorithm}

The map matching process is to find within the digital map the lane markings that are observed by the camera. The algorithm presented in this paper combines both the vehicle pose, the protection levels associated with a given risk $\alpha$, the measurements of the camera with their uncertainties and the high definition map with positional uncertainties.

For each camera measurement, and with the use of the protection levels and the camera uncertainty, the method generates a search area centered on the estimated detected marking. This area represents the possible locations of the point $L$ representing the detected marking. Therefore, any marking from the map intersecting this area is considered as a plausible candidate for the map-matching. It is possible to have several markings intersecting this area and so several candidates. However, with the combination of the different camera measurements and the road configuration (one, two or more lanes), it is possible in some cases, to disambiguate the situation and have a final unique matching hypothesis for each detected marking.

\section{B. Search polygon}

The first step of the algorithm consists in defining the search area $S_{T I R}$ for a given TIR (Fig. 3). This area has to take into account uncertainties on both the pose $\left(P L_{x}\right.$, $\left.P L_{y}, P L_{\theta}\right)$ and the camera measurement $\left(\Delta C_{0}\right)$. Computing directly this shape might be tricky so the construction is divided into three steps.

- Step 1: we first ignore $P L_{\theta}$. Due to $P L_{x}$ and $P L_{y}$, the actual position of point $M$ may be anywhere within a $2 \cdot P L_{x}$ length and $2 \cdot P L_{y}$ width rectangle. As a result, the detected lane marking point might be anywhere in this same size rectangle but translated to point $L$ (on Fig. 3-a: plain black rectangle). The camera measurement is also uncertain, therefore the rectangle needs to be augmented by $2 \cdot \Delta C_{0}$ along the lateral axis. We obtain a $2 \cdot\left(P L_{y}+\Delta C_{0}\right) \times 2 \cdot P L_{x}$ rectangle centered on $\mathrm{L}$ (red colored area in Fig. 3-a).

- Step 2: we now introduce $P L_{\theta}$. If the heading of the vehicle belongs to $\left[\theta-P L_{\theta} ; \theta+P L_{\theta}\right]$, the origin of $R_{C}$ is moving on an arc and so is the previously defined rectangle. The domain covered by the rectangle when the heading $\theta$ goes from $\theta-P L_{\theta}$ up to $\theta+P L_{\theta}$ is a shape with two curved sides. There is an outer convex side (between points $B$ and $C$ in Fig. 3-b) and an inner concave side (between points $E$ and $F$ in Fig. 3-b).

- Step 3: having to manipulate the search domain resulted from Step 2 is not convenient because of the curved parts, therefore, we will over-bound it by a convex polygonal hull. Because the inner side in concave, we link the points $E$ and $F$ by a straight line. On the contrary, because the outer side in convex, a straight line between the points $B$ and $C$ will not lead to an over-bounding. Instead, we add an additional point $G$ with corresponds to the intersection of the tangent at point $B$ and the tangent at point $C$. The final search domain (see Fig. 3-c) is a convex polygon containing the one from the previous step, therefore verifying the integrity constraint.

\section{Map-matching hypothesis}

A lane marking is also represented by a polygon, therefore a lane marking is considered as a candidate for the mapmatching is its corresponding polygon intersects the search polygon defined previously. To find candidate markings, we can use a clipping algorithm such as Weiler-Atherton [15] or Sutherland-Hodgman [11] algorithms. We used the later one as it is optimized for convex polygons. These algorithms return the intersection between two polygons. If the result is not empty then the marking is considered as a plausible hypothesis. Only nearby markings are treated to optimize the algorithm. The map contains a R-tree index to efficiently retrieve markings in a rectangular area.

Depending on the considered target integrity risk, there could be no matching (Fig. 4-a), a unique matching (Fig. 4b) or multiple matching candidates (Fig. 4-c). It is important to note that as we have supposed the map error to be strictly bounded, the bounds on the lane markings do not change w.r.t. the TIR. On the contrary, a high TIR will result in a small search polygon while a low TIR will lead to a large one.

The optimal strategy of the algorithm is to give an integrity risk range in which the map matching is not ambiguous. However, determining this range, even with a dichotomous method has a high computational cost. The solution retained to keep a low computation cost, to the detriment of the algorithm availability, is to execute the algorithm with a preselected TIR scale to roughly constrict the true range.

\section{Disambiguation with multiple measurements}

Multiple marking hypotheses could result from very large protection levels or large uncertainties. However, it is possible to disambiguate the hypotheses if the road lanes configuration is jointly used with several camera measurements. 


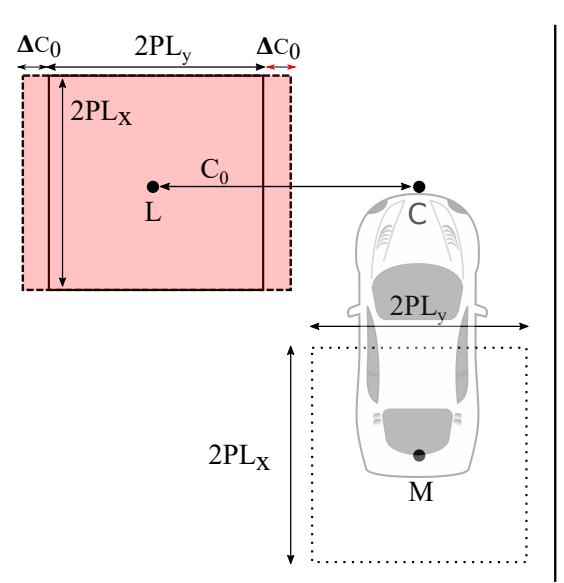

(a) Step 1

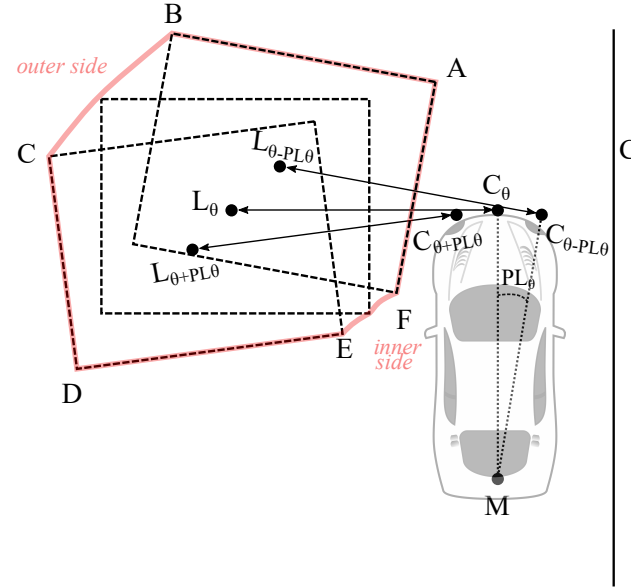

(b) Step 2

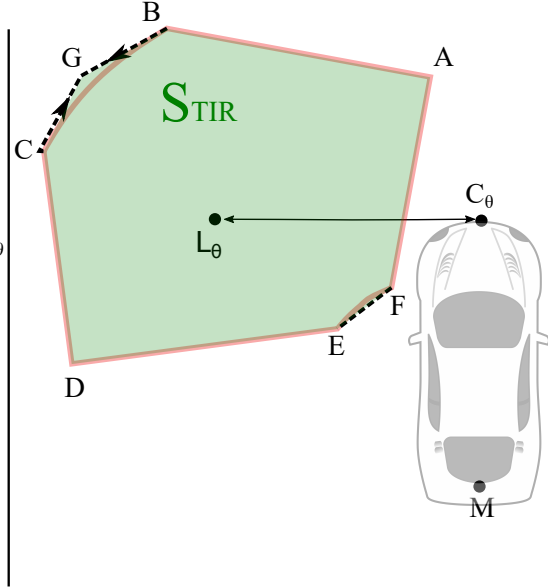

(c) Step 3

Fig. 3. Steps of the search area construction. Step 1: without using $P L_{\theta}$. Step 2: introducing $P L_{\theta}$ to compute intermediate areas. Step 3: final area (green) over-bounding previous areas of step 2.

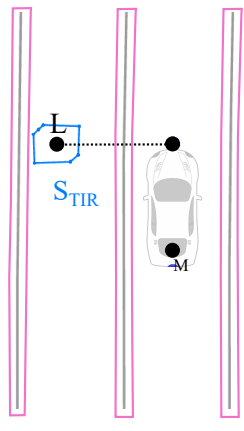

(a)

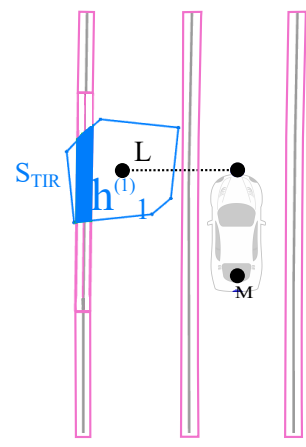

(b)

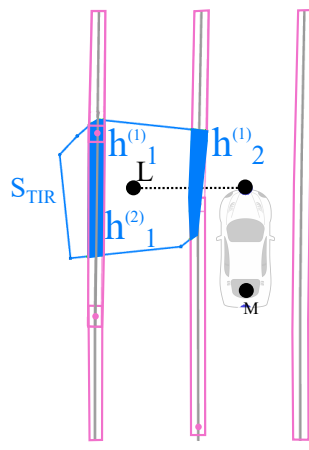

(c)

Fig. 4. (a): No map-matching because of small PL (high TIR). (b): one non-ambiguous map-matching hypothesis. (c) : three map-matching hypotheses because of too large PL (low TIR). Search area is in blue $\left(S_{T I R}\right)$, the intersection between search area and marking is plain blue. $h_{i}^{(k)}$ is the hypothesis of matching $k$-th segment of the $i$-th marking.

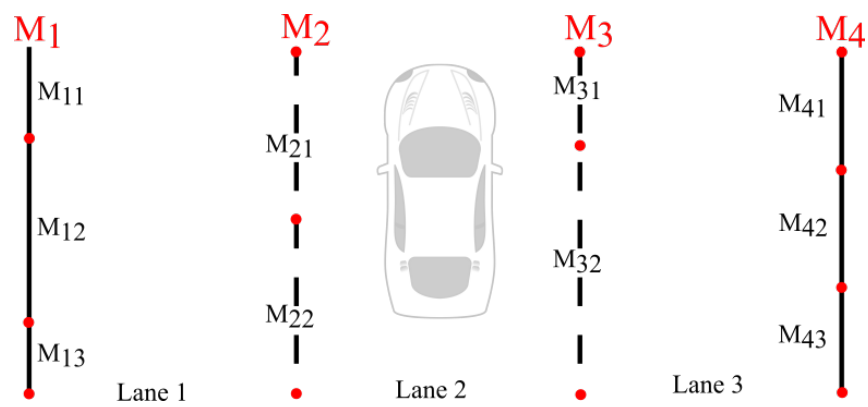

Fig. 5. Road configuration with three lanes and four lane markings $M_{i}$, $i \in\{1,2,3,4\} . M_{i j}$ is the $j$-th segment of the $i$-th marking.

We remind that lane markings are made up of a sequence of line segments. We introduce the following notations: $M_{i j}$ represents the $j$-th segment of the $i$-th marking of the map, $(i, j) \in \mathbb{N}^{2}$ and $h_{i}^{(k)}$ is the $k$-th map-matched marking hypothesis corresponding to the $i$-th camera measurement, $i \in\{1,2,3,4\}$. The marking segments are ordered from left to right (according to $R_{C}$ ) so the index $i$ of $M_{i}$ represents the $i$-th marking starting from the left. Moreover, only markings nearby the car are considered. We also consider that all connected segments represent the same marking and thus the same hypothesis. The $h_{i}^{(k)}$ hypotheses are merged:

$$
\forall i \in \mathbb{N}, H_{i}=\left\{h_{i}^{(k)}, \forall k \in \mathbb{N}\right\} .
$$

The disambiguation process is based on the assumption that the car is always driving on a lane and not outside of the road; thus left side detected markings cannot be matched with the most right road marking on the map and vice versa:

$$
\left(H_{4 \vee 3} \neq M_{1}\right) \wedge\left(H_{1 \vee 2} \neq M_{n}\right),
$$

with $M_{n}$ the most right markings given the context. The camera also never returns two measurements for the same detected marking:

$$
\forall(i, j) \in \mathbb{N}^{2}, i \neq j \Rightarrow H_{i} \neq H_{j} .
$$

The camera measurements are ordered, from left to right, which implies:

$$
\left(H_{m}=M_{i}\right) \&\left(H_{n}=M_{j}\right) \Rightarrow m<n \& i<j .
$$

The algorithm generates all valid combinations from the map matched hypotheses. To do so, a depth-first possibility 
tree exploration is made. First, some hypotheses are united according to (5). Then, at each node, conditions (6), (7) and (8) must be verified; if not the exploration of the branch is abandoned. If there is only one hypothesis left, then all ambiguities are removed and the final map-matching is returned.

For instance, let us consider the configuration as pictured in Fig. 5: a three-lane road delimited by four lane markings. Suppose that the camera provides four marking detections and that the map matching algorithm returned the following hypotheses :

$$
\begin{array}{ll}
\text { - } & H_{1}=\left\{\left(M_{11} \vee M_{12}\right) \vee\left(M_{21}\right)\right\}=M_{1} \vee M_{2} \\
\text { - } & H_{2}=\left\{\left(M_{11}\right) \vee\left(M_{21} \vee M_{22}\right) \vee\left(M_{31}\right) \vee\left(M_{41}\right)\right\}= \\
& M_{1} \vee M_{2} \vee M_{3} \vee M_{4} \\
\text { - } & H_{3}=\left\{\left(M_{21}\right) \vee\left(M_{31}\right) \vee\left(M_{41} \vee M_{42}\right)\right\}=M_{2} \vee M_{3} \vee M_{4} \\
\text { - } & H_{4}=\left\{\left(M_{31}\right) \vee\left(M_{41} \vee M_{42}\right)\right\}=M_{3} \vee M_{4}
\end{array}
$$

Rule (5) merges segments of the same marking. If rule (6) is applied, then $M_{4}$ is removed from $H_{2}$ because the most right marking cannot be matched with a left side measurement. If we suppose that $H_{1}=M_{2}$ then because of rules (7) and (8), we would get $H_{2}=M_{3}, H_{3}=M_{4}$ and $H_{4}=\emptyset$, which is impossible. If we keep applying rules, we finally obtain one unique $M_{i}$ marking for each hypothesis $H_{j}$, which means there is no more ambiguities: $\left(H_{1}=M_{1}\right) \wedge\left(H_{2}=M_{2}\right) \wedge$ $\left(H_{3}=M_{3}\right) \wedge\left(H_{4}=M_{4}\right)$.

\section{E. Constraints on detected markings}

The camera provides additional information about detected markings: the type of marking (dashed, solid, double, etc.) and the quality of the detection (with value in $\{0,1,2,3\}$ ). It is possible to add constraints to the map-matching algorithm and decide to ignore the low quality detected markings (i.e., 0 or 1 , which represent about $20 \%$ of the measurements) or ignore markings from the map if the detected type does not match. We will measure the impact of these restrictions in the experimental part.

\section{EXPERIMENTS}

The algorithm has been tested with real data recorded on open road using ROS framework. The vehicle was equipped with a RTK-GNSS receiver to provide the ground truth localization. A Mobileye camera was used to provide a detection of up to four lane markings at a time. The experiment took place in December 2017 in Palaiseau, France (Fig. 6). The map-matching algorithm has been implemented in Python and runs in real time at $30 \mathrm{~Hz}$.

\section{A. System availability}

We first set the TIR to $10^{-4}$ and assessed the localization system availability w.r.t. to the level of positioning error. We added artificial noise to the position data to simulate errors in the pose estimation. A Gaussian noise $\mathcal{N}(0, \Sigma)$ was added to the RTK-GNSS measurements, which are considered as the ground truth. The over-bound $\Delta C_{0}$ of the camera measurement errors and the radius $l$ of the map errors were set to $60 \mathrm{~cm}$.
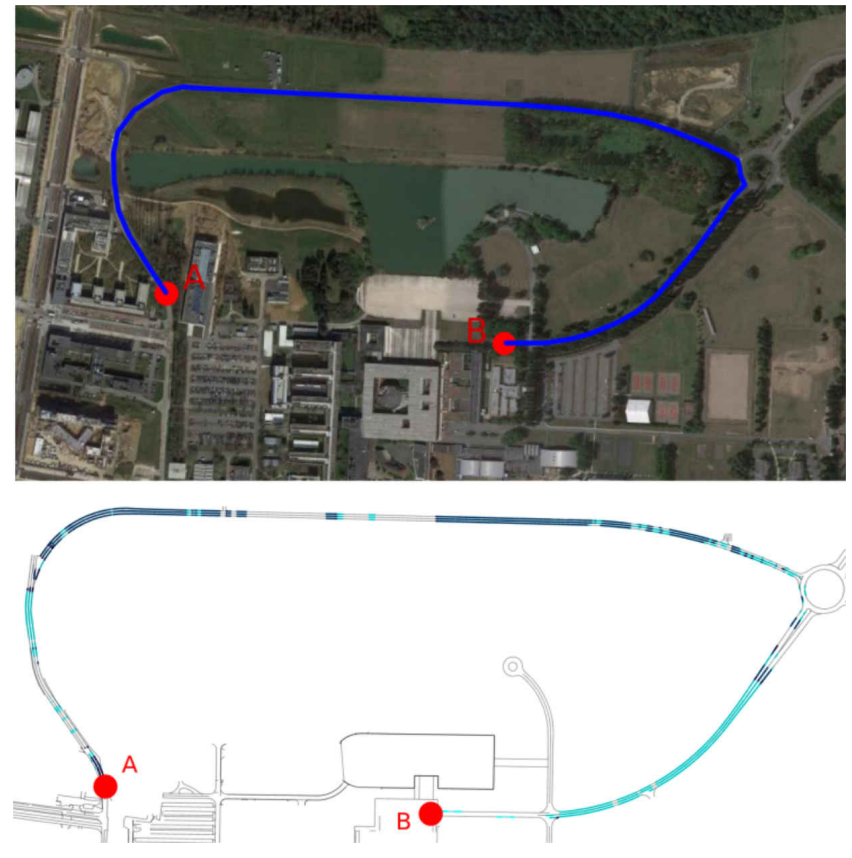

Fig. 6. Top: overview of the test site with the trajectory from point A to B. Bottom : colored map-matched marking. Matched markings with restrictions on quality levels and marking types are in dark blue and matched markings without restriction on quality or type are in light blue.

Figure 7 shows the system availability with respect to the noise standard deviation. In the case where only GNSS pose was used, the system was considered as available when the pose and its protection levels remained inside the lane. With the use of the camera, the system was considered as available when the map-matching algorithm returned a nonambiguous hypothesis. The system availability was computed with variants of our algorithm: we decided alternatively to ignore or not the types and the quality level of the detected markings.

Unsurprisingly, the availability of the GNSS only solution decreases very quickly when the noise variance increases. Also, removing lowest quality detections and forcing correspondence of marking type at the same time gave the lowest rates. The version of the algorithm where marking types were handled regardless of the quality gave the best results. Indeed, keeping only highest detection qualities could lead to no measurements at all or to a lack of measurements and realizing disambiguation would be impossible. On the other hand, keeping all quality measurements and not requiring a good correspondence of marking types leads to multiple map-matching hypotheses which are impossible to disambiguate.

\section{B. Integrity risk}

Conversely, we then set the variance to fixed value $\sigma^{2}=0.75$, and studied the minimum level of integrity risk achievable using either the GNSS only solution or the map-matching one. We used our map-matching algorithm with marking type correspondences as it has been shown previously to have the highest availability. Figure 8 shows 


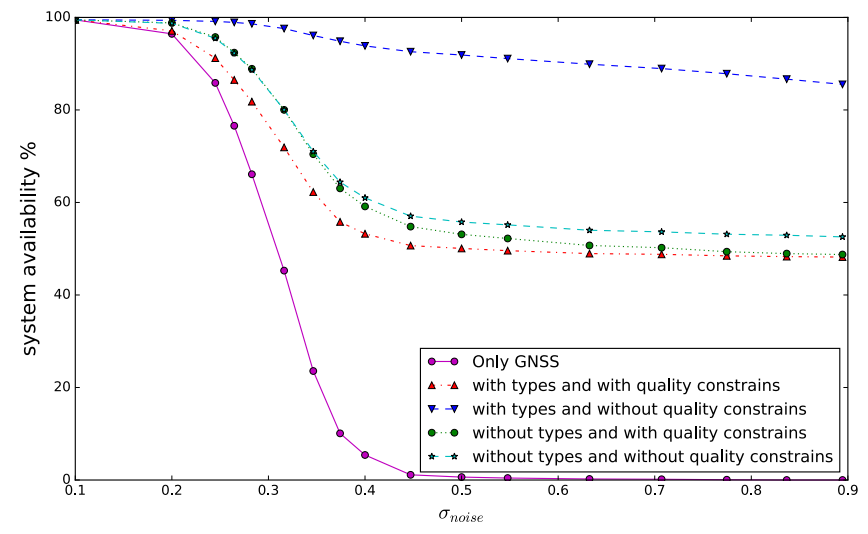

Fig. 7. System availability with $\mathrm{TIR}=10^{-4}$.

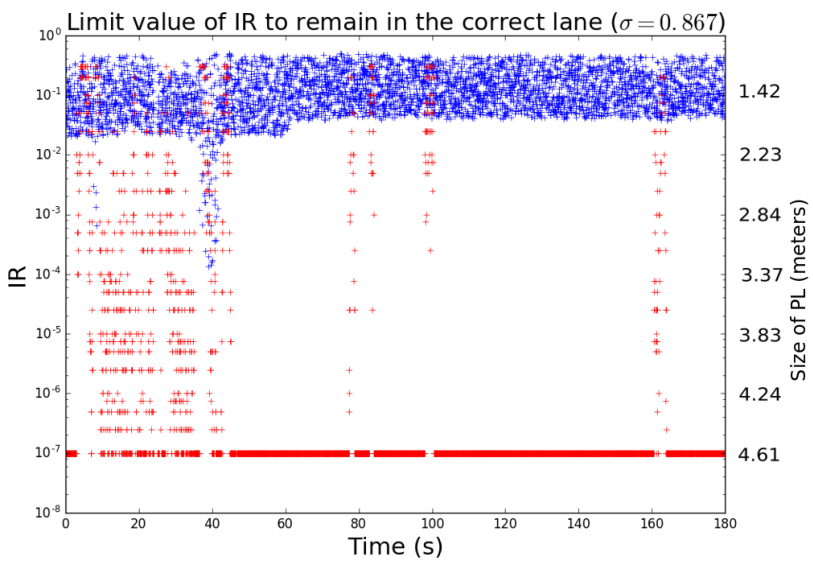

Fig. 8. Limit value of Integrity Risk to remain in the correct lane $(\sigma=$ 0.867), blue points: the GNSS only solution, red points: the proposed mapmatching solution. The lowest Integrity Risk computed is $10^{-7}$.

the limit integrity risk with respect to the time throughout the experiment. Blue points were computed with the GNSS only solution and red ones with the algorithm we proposed. The lowest Integrity Risk used was $10^{-7}$, as a consequence true limit could be lower. Even with a very low integrity risk our map-matching solution was able to give non-ambiguous results; the 90 -th percentile is $10^{-4}$. On the contrary, the GNSS only solution median is 0.18 which shows that integrity would not be respected most of the time even if a high risk is set, typically 0.05 .

\section{CONCLUSIONS}

In this paper, a new method for lane level localization has been presented which aims at providing results with high integrity. This method combines pose protection levels and over-bounding of errors when the underlying distributions are unknown. The algorithm advantages lie in its efficiency and its ability to give reliable lane level localization, with an IR as low as $10^{-7}$, while keeping a high availability. The algorithm efficiency relies on the basic idea of using polygon intersections to express hypotheses. Furthermore, the algorithm is simple and easy to implement and contrary to classical geometrical approaches, the algorithm is insensitive to map imprecision.

The output of the map-matching can further serve as an input to a localization estimator in order to improve the pose estimation itself. One issue that was not considered in this paper is the case of missing markings in the map. The proposed algorithm may not be resilient to such a problem, especially when only few detected markings are available. One possible solution to alleviate such an issue would be to consider temporal consistency in the map-matching process.

\section{REFERENCES}

[1] F. Abdallah, G. Nassreddine, and T. Denoeux. A multiple-hypothesis map-matching method suitable for weighted and box-shaped state estimation for localization. IEEE Transactions on Intelligent Transportation Systems, 12(4):1495-1510, Dec 2011.

[2] G. Agamennoni, J. I. Nieto, and E. M. Nebot. An outlier-robust kalman filter. In Proceedings of the IEEE International Conference on Robotics and Automation, pages 1551-1558, Shanghai, China, May 2011.

[3] $\mathrm{Ph}$. Bender, J. Ziegler, and Ch. Stiller. Lanelets: Efficient map representation for autonomous driving. In Proceedings of IEEE Intelligent Vehicles Symposium, pages 420-425, Ypsilanti, MI, USA, 2014.

[4] D. Bétaille and R. Toledo-Moreo. Creating enhanced maps for lanelevel vehicle navigation. IEEE Transactions on Intelligent Transportation Systems, 11(4):786-798, Dec 2010.

[5] K. A. Bin Ahmad, M. Sahmoudi, and Ch. Macabiau. Characterization of GNSS Receiver Position Errors for User Integrity Monitoring in Urban Environments. In European Navigation Conference, Rotterdam, Netherlands, April 2014.

[6] H. Dbouk and S. Schön. Comparison of different bounding methods for providing gps integrity information. In Proceedings of IEEE/ION Position, Location and Navigation Symposium, pages 355-366, Monterey, CA, USA, April 2018.

[7] F. Li, Ph. Bonnifait, and J. Ibañez-Guzmán. Using High Definition Maps to Estimate GNSS Positioning Uncertainty. In European Navigation Conference, Lausanne, Switzerland, May 2017.

[8] M. E. B. El Najjar and Ph. Bonnifait. Road selection using multicriteria fusion for the road-matching problem. IEEE Transactions on Intelligent Transportation Systems, 8(2):279-291, June 2007.

[9] National Imagery and Mapping Agency. Department of defense world geodetic system 1984: its definition and relationships with local geodetic systems. Technical Report TR8350.2, St. Louis, MO, USA January 2000.

[10] P. F. Navarro Madrid, M. D. Laínez Samper, and M. M. Romay Merino. New approach for integrity bounds computation applied to advanced precise positioning applicationsg. In Proceedings of the 28th International Technical Meeting of The Satellite Division of the Institute of Navigation, pages 2821-2834, Tampa, FL, USA, September 2015

[11] I. E. Sutherland and G. W. Hodgman. Reentrant polygon clipping. Communications of the ACM, 17(1):32-42, January 1974

[12] Z. Tao and Ph. Bonnifait. Sequential data fusion of GNSS pseudoranges and Dopplers with map-based vision systems. IEEE Transactions on Intelligent Vehicles, 1(3):254-265, Sep. 2016.

[13] Z. Tao, Ph. Bonnifait, V. Frémont, and J. Ibañez-Guzmán. Lane marking aided vehicle localization. In Proceedings of the 16th International IEEE Conference on Intelligent Transportation Systems, pages 1509-1515, The Hague, Netherlands, Oct 2013.

[14] T. A. Tran, C. Jauberthie, F. Le Gall, and L. Travé-Massuyès. Interval kalman filter enhanced by positive definite upper bounds. IFAC Proceedings Volumes, 50(1):1595 - 1600, 2017.

[15] K. Weiler and P. Atherton. Hidden surface removal using polygon area sorting. In Proceedings of the 4th annual conference on Computer graphics and interactive techniques, pages 214-222, San Jose, CA, USA, Jul 1977.

[16] Ch. E. White, D. Bernstein, and A. L. Kornhauser. Some map matching algorithms for personal navigation assistants. Transportation Research Part C: Emerging Technologies, 8(1):91 - 108, 2000. 